\title{
Molecular biochemical characterization of selective glucocorticoid receptor activities of GSK866 analogues with cysteine reactive warheads
}

Wim Vanden Berghe ${ }^{1}$, Chandra S. Chirumamilla ${ }^{1}$, Ajay Palagani ${ }^{1,2}$, Marinus Verbeeck ${ }^{1}$, Ryabtsova Oksana ${ }^{3}$, Balu Kamaraj ${ }^{4}$, Karolien De Bosscher ${ }^{5}$, Nadia Bougarne ${ }^{5}$, Bart Ruttens ${ }^{6}$, Kris Gevaert ${ }^{6}$, Rene Houtman ${ }^{7}$, Winnok H. De Vos ${ }^{8}$, Jurgen Joossens ${ }^{3}$, Pieter Van Der Veken ${ }^{3}$, Koen Augustyns ${ }^{3}$, Xaveer Van Ostade ${ }^{1}$, Annemie Bogaerts ${ }^{4}$, Hans De Winter ${ }^{3}$

${ }^{1}$ Laboratory of Protein Chemistry, Proteomics and Epigenetic Signalling, Department of Biomedical Sciences, University of Antwerp (UA), Belgium, ${ }^{2}$ Radiation Oncology Department, Sidney Kimmel Medical College of Thomas Jefferson University, Philadelphia, PA, USA, ${ }^{3}$ Medicinal Chemistry, Department of Pharmaceutical Sciences, University of Antwerp, Universiteitsplein 1, B-2610 Antwerp, Belgium, ${ }^{4}$ Research group PLASMANT, Department of Chemistry, University of Antwerp, Universiteitsplein 1, 2610, Wilrijk-Antwerp, Belgium, ${ }^{5}$ Nuclear Receptor Signaling Unit, CRL, VIB-UGent Department of Medical Protein Research, Ghent, Belgium, ${ }^{6}$ Center for Medical Biotechnology, VIB-UGent Department of Biochemistry, Ghent, Belgium, ${ }^{7}$ PamGene International B.V., Den Bosch, the Netherlands,

Synthetic glucocorticoids (GC) are the mainstay therapy for treatment of acute and chronic inflammatory disorders. Due to the high adverse effects associated with long-term use, GC pharmacology has focused since the nineties on more selective glucocorticoid ligand binding strategies, classified as selective glucocorticoid receptor agonists (SEGRAs) or selective glucocorticoid receptor modulators (SEGRMs). Following molecular modeling, a novel series of GSK866 SEGRA analogues with covalent warheads was designed and synthesized, analogous to the concept of selective covalent binding kinase inhibitors. Since the off-rate of a covalently binding drug is negligible compared to that of a non-covalent drug, its therapeutic effects can be prolonged and typically, smaller doses of the drug are necessary to reach the same level of therapeutic efficacy, thereby potentially reducing systemic side effects. Different SEGRA GSK866analogues with cysteine reactive warheads were characterized for GR potency and selectivity in various biochemical and cellular assays. GR- and $\mathrm{NF} \kappa \mathrm{B}$-dependent reporter gene studies show favorable anti-inflammatory properties with reduced GR transactivation of two non-steroidal GSK866 analogues UAMC-1217 and UAMC-1218, whereas UAMC-1158 and UAMC-1159 compounds failed to modulate cellular GR activity. These results were further supported by GR immuno-localization and S211 phospho-GR western analysis, illustrating significant GR phosphoactivation and nuclear translocation upon treatment of GSK866, UAMC-1217 or UAMC-1218, but not in case of UAMC-1158 or UAMC-1159. Furthermore, mass spectrometry analysis of tryptic peptides of recombinant GR-LBD bound to UAMC-1217 or UAMC-1218 confirmed covalent cysteinedependent GR binding. Finally, molecular dynamics simulations, as well as GR-LBD coregulator interaction profiling of the GR-LBD bound to GSK866 or its covalently binding analogues UAMC-1217 or UAMC1218 revealed subtle conformational differences that might underlie their SEGRA properties. Altogether, GSK866 analogues 1217 and 1218 hold promise as a novel class of covalent binding SEGRA ligands for the treatment of inflammatory disorders. 